\title{
A GRAPHICAL METHOD OF DETERMINING THE P CORRECTION
} IN THE DEFLECTION EXPERIMENT.

By W. Watson.

Some time ago, in connection with some experiments on the question of suitable relation between the deflecting and the deflected magnets for use in the measurement of $H$, it was necessary to calculate the value of the $P$ corrections for a number of observations. This calculation, if we adopt the ordinary expression, or even the approximate one given by Ricker and Thorpe,' takes some little time, and the following graphical method was devised. Although it is so simple that it is very unlikely that it has not already been used, yet, since I never remember having seen it described, it may be of some interest to readers of "Terrestrial Magnetism."

The expression connecting the magnetic moment $M$ of the deflecting magnet when placed at a distance $r$, the value of $H$ and the angle

$\theta$ through which the instrument is turned nay be put in the following form:

$$
\frac{H}{M}=\frac{2}{r^{3}}\left(\operatorname{cosec} \theta\left(\mathrm{I}-g t-q^{\prime} t^{2}-\frac{2 \mu}{r^{3}}\right)\left(\mathrm{r}+\frac{P}{r^{2}}\right)\right.
$$

in which $t$ is the temperature reckoned from some standard temperature, $q$ and $q^{\prime}$ are, respectively, the first and second temperature coefficients of the deflecting magnet, $\mu$, the induction coefficient, and $P$ the distribution coefficient, the relative dimensions of the deflecting and deflected magnet being such as to make the succeeding distribution coefficients, $Q, R$, etc., negligible.

However, for simplicity of calculation, it is usual to make use of the approximation

so that

$$
\left(\mathrm{I}+\frac{P}{r^{2}}\right)^{-1}=\mathrm{I}-\frac{P}{r^{2}},
$$

$$
\frac{H}{M}\left(\mathrm{I}-\frac{P}{r^{2}}\right)=\frac{2}{r^{8}} \operatorname{cosec} \theta\left(\mathrm{I}-q t-q^{\prime} t^{2}-\frac{2 \mu}{r^{3}}\right)
$$

In obtaining this expression, powers of $\frac{P}{r}$ above the first have been neglected, and hence it is useless to employ a formula for deducing $P$ which takes account of higher powers of $\frac{P}{r^{2}}$ than the first.

Now if $A$ and $A_{1}$ are the reciprocal values of the right hand side of equation ( $I$ ) above for two values of $r$ and $r_{1}, r$ being the shorter distance, the ordinary expression for calculating $P$ is:

$$
\frac{A}{A_{1}}=\frac{\mathrm{I}-\frac{r}{r_{1}^{2}}}{\mathrm{I}-\frac{\mu^{\prime \prime}}{r^{-x}}}
$$

1 Phil. Trans. I889 A, p. 7 I. 
Hence,

$$
\begin{aligned}
\log _{10} A-\log _{10} A_{1} & =\log _{10} e\left[\log \left(\mathrm{I}-\frac{P}{r_{1}^{2}}\right)-\log _{e}\left(\mathrm{r}-\frac{P}{r^{2}}\right)\right] \\
& =\log _{10} e \cdot P\left(\frac{\mathrm{I}}{r^{2}}-\frac{\mathrm{I}}{r_{1}^{2}}\right) .
\end{aligned}
$$

in which we have neglected powers of $\frac{P}{r^{2}}$ above the first.

Or writing the result thus:

$$
\frac{\log _{10} A-\log _{10} A_{1}}{P}=\log _{10} e\left(\frac{\mathrm{I}}{r^{2}}-\frac{\mathrm{I}}{r_{1}^{2}}\right)
$$

That is, if the two distances at which the observations are made are kept constant, so that $\frac{r}{r^{2}}-\frac{r}{r_{1}{ }^{2}}$ is constant, we have that there is a linear relation between $\left(\log A-\log A_{1}\right)$ and $P$, so that if by any method we calculate a value of $P$, which gives the correct value for the expression $\left(\log A-\log A_{1}\right)$, then the value so obtained must be the correct one.

Suppose now we plot a curve in which the ordinates are the values of $P$, and the abscissae the corresponding values of $\left(\log A-\log A_{1}\right)$. Since (2) shows that this curve will be a straight line, it will only be necessary to calculate two points, or, if we make use of the fact that when $P$ is zero ( $\log A-$ $\log A_{1}$ ) is also zero, only one point. This curve which is represented by the straight line $O A$ in the figure will enable us to immediately find $P$ when we know the value of the difference between the values of $\log A$, which is obtained in the process of reducing the observations.

What is really required in reducing the observations for the value of $H$ is not, however, $P$, but the values of $\log \left(\mathrm{I}-P / r^{2}\right)$ for the two distances employed. Now to the approximation we are using, we have

$$
\log _{10}\left(\mathrm{I}-\begin{array}{c}
P \\
r^{2}
\end{array}\right)=-\log _{10} e \frac{P}{r^{2}}=C P,
$$

where $c$ is a constant for any particular value of $r$-thus there is a linear relation between $\log \left(\mathrm{x}-P / r^{2}\right)$ and $P$. Hence we may draw a straight line to represent the connection between these two quantities. This line is represented in the figure by $B D$ for $r=30 \mathrm{~cm}$., and by $C D$ for $r_{1}=40 \mathrm{~cm}$. Thus by means of these two lines we can immediately find the values of $\log \left(1-P / 30^{2}\right)$ and $\log \left(\mathrm{I}-P / 40^{2}\right)$ if we know the value of $P$, this value being obtained from the line $O A$.

By drawing these lines on a sheet of squared paper, the distance between the parallel lines being a lenth of an inch, and taking one inch to represent one unit in $P$ and 50 units in the fifth place of decimals in the logarithms, it is quite easy to read off the values of the quantities $\log \left(I-R / 30^{2}\right)$ and $\log$ (I $\left.-P / 40^{\circ}\right)$ correct to a unit in the fifth place. 


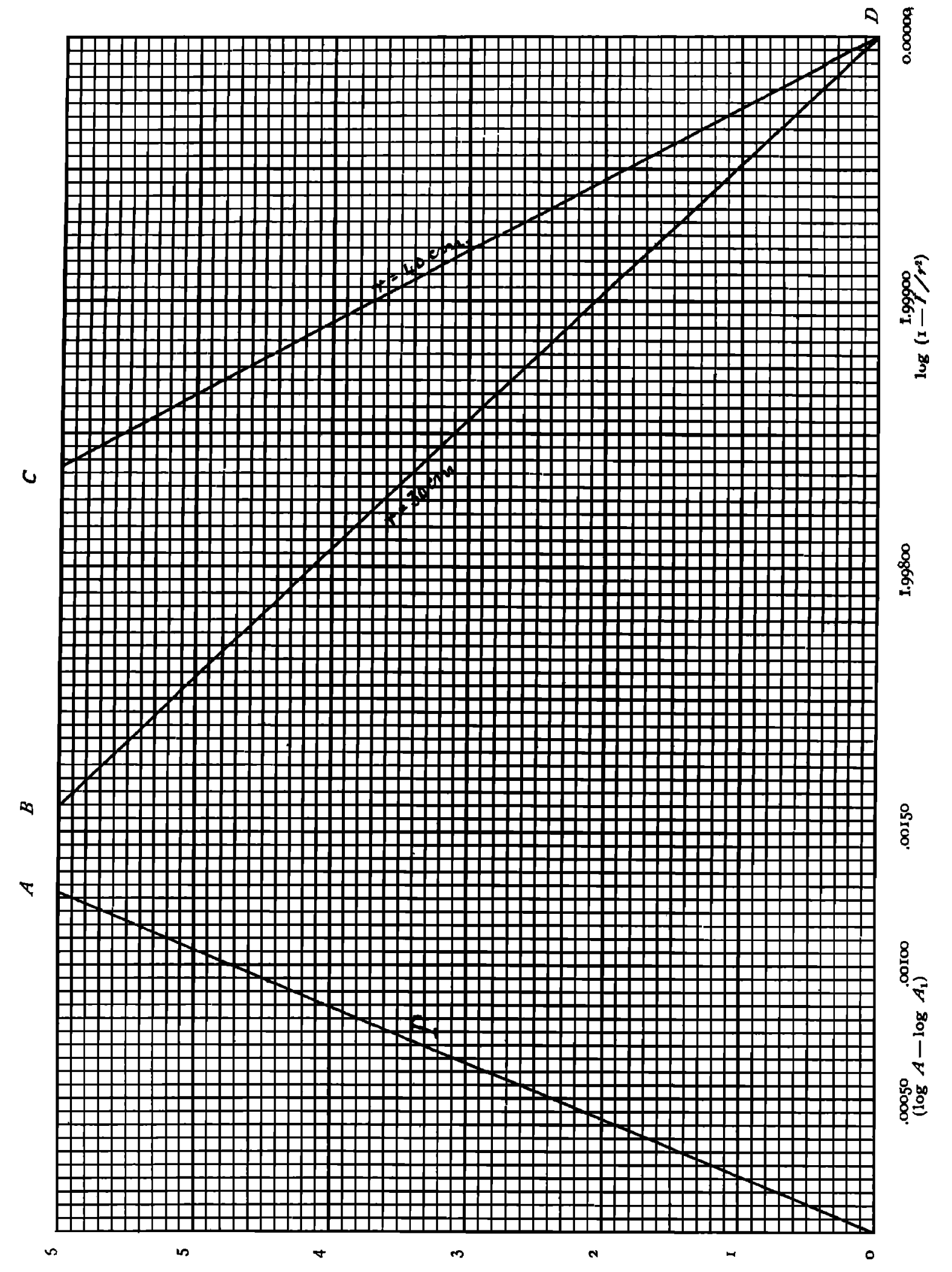

